\title{
Obesity is associated with AKI after surgery via oxidative stress
}

Investigators at Vanderbilt University School of Medicine suggest that obesity is an independent risk factor for acute kidney injury (AKI) after cardiac surgery, and that this association is partially mediated by oxidative stress. "As the prevalence of obesity continues to increase in cardiac surgery cohorts, we conducted this study to address three questions", says researcher Frederic T. Billings, IV. "First, does obesity predict kidney injury following cardiac surgery; second, do intraoperative markers of oxidative stress, inflammation or antifibrinolysis predict postoperative kidney injury; and third, do these candidate pathways mediate any

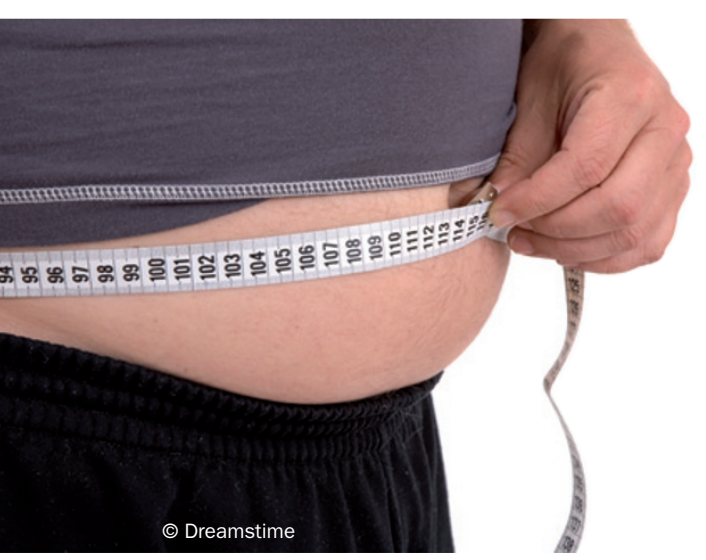

identified association between obesity and kidney injury."

Of 445 patients included in the study, $25.2 \%$ developed stage I AKI after cardiac surgery, defined as a $\geq 26.5 \mu \mathrm{mol} / \mathrm{l}$ or $50 \%$ increase in serum creatinine concentration within $72 \mathrm{~h}$ of surgery. Patients who developed AKI had a longer hospital stay, were more likely to develop postoperative atrial fibrillation, were more likely to develop pneumonia and had a higher risk of death at 30 days than did patients who did not develop AKI. After adjustment for baseline risk factors for AKI, the odds of AKI increased by $26.5 \%$ per $5 \mathrm{~kg} / \mathrm{m}^{2}$ increase in BMI.

When the investigators measured markers of oxidative stress, antifibrinolysis and inflammation, they found that levels of $\mathrm{F}_{2}$-isoprostanes were significantly higher at baseline and during surgery in patients who developed AKI than in those who did not develop AKI. By contrast, baseline levels of IL-6 and PAI-1 did not predict AKI although intraoperative concentrations of PAI-1 were higher in patients who developed AKI. For any given $\mathrm{BMI}$, intraoperative levels of $\mathrm{F}_{2}$-isoprostanes were higher in patients with AKI than in those without AKI. BMI no longer predicted AKI after adjustment for the effect of $\mathrm{F}_{2}$-isoprostanes, whereas IL-6 and PAI-1 did not alter the association between BMI and AKI. Relationships between BMI, candidate pathway biomarkers and AKI were examined by deconstructing the total effect of BMI on AKI into direct effects and indirect effects. "Our data suggest that the association between obesity and kidney injury is partially mediated by the effect of BMI on oxidative stress but not inflammation or antifibrinolysis", comments Billings.

The authors suggest that interventions that reduce the formation of $\mathrm{F}_{2}$-isoprostanes could potentially reduce the risk of AKI, particularly in obese patients. "Physicians should consider performing clinical trials of therapies that may reduce oxidative stress during surgery and measure the effect of these therapies on intraoperative plasma concentrations of $\mathrm{F}_{2}$-isoprostanes", says Billings. "By doing so, we may develop therapies that reduce the incidence of kidney injury following cardiac surgery."

Helene Myrvang

Original article Billings, F. T. et al. Obesity and oxidative stress predict AKI after cardiac surgery. J.Am. Soc. Nephrol. doi:10.1681/ASN.2011090940 